CARDIOVASCULAR MEDICINE

\title{
Relation between left ventricular contractile reserve during low dose dobutamine echocardiography and plasma concentrations of natriuretic peptides
}

\author{
A F L Schinkel, E C Vourvouri, J J Bax, F Boomsma, M Bountioukos, V Rizzello, E Biagini, \\ E Agricola, A Elhendy, J R T C Roelandt, D Poldermans
}

Heart 2004;90:293-296. doi: 10.1136/hrt.2002.003822

See end of article for authors' affiliations

.....................

Correspondence to: Dr Don Poldermans, Thoraxcentre Room $\mathrm{Ba}$ 300, Department of Cardiology, Erasmus Medical Centre, $\mathrm{Dr}$ Molewaterplein 40, 3015 GD Rotterdam,

Netherlands;

d.poldermans@

erasmusmc.nl

Accepted 17 June 2003

\begin{abstract}
Background: In ischaemic cardiomyopathy, raised plasma concentrations of natriuretic peptides are associated with a poor long term prognosis, while the presence of contractile reserve is a favourable sign. Objective: To assess the relation between plasma natriuretic peptides and contractile reserve.

Design: Prospective observational study.

Setting: Tertiary referral centre.

Patients: 66 consecutive patients undergoing low dose dobutamine stress echocardiography to evaluate contractile reserve in regions with contractile dysfunction at rest, divided into two groups: group 1, 31 patients with ischaemic cardiomyopathy (left ventricular ejection fraction $\leqslant 40 \%$ ) and heart failure symptoms; group 2, 35 patients with normal left ventricular function.

Main outcome measures: Plasma atrial natriuretic peptide (ANP) and brain natriuretic peptide (BNP), measured using immunoradiometric assays. Contractile reserve was defined as an improvement in segmental wall motion score during infusion of low dose dobutamine.

Results: Plasma ANP and BNP concentrations were higher in group 1 than in group 2 (mean (SD): ANP, 17.8 (32.8) v 7.2 (9.7), $p<0.005 ; B N P, 24.4$ (69.0) v 5.0 (14.3) pmol/I, respectively; $p<0.001$ ). In group 1, the presence of contractile reserve was inversely related to ANP and BNP levels; however, patients with contractile reserve had lower ANP and BNP concentrations than patients without contractile reserve (ANP, $14.2(9.1) \vee 24.2(44.2), \mathrm{p}<0.05 ; \mathrm{BNP}, 20.2(25.5) \vee 37.5(93.8) \mathrm{pmol} / \mathrm{l}$, respectively; $\mathrm{p}<0.05)$.

Conclusions: Plasma natriuretic peptide concentrations are raised in patients with left ventricular dysfunction, but in the presence of preserved myocardial contractile reserve, relatively low levels of ANP and $B N P$ are present.
\end{abstract}

o schaemic left ventricular dysfunction is the principal cause of congestive heart failure, which has a poor long term prognosis. ${ }^{1-3}$ It has recently been proposed that atrial natriuretic peptide (ANP) and brain natriuretic peptide (BNP) may be useful in evaluating outcome in patients with heart failure. ${ }^{4-8}$ ANP is a cardiac hormone which is synthesised and secreted primarily in the atria, whereas $\mathrm{BNP}$ is produced in the ventricles in response to changes in wall stretch. Plasma concentrations of these natriuretic peptides are raised in patients with left ventricular dysfunction, which is associated with a poor long term prognosis. ${ }^{4-8}$

In patients with depressed left ventricular function, the presence of improved function during dobutamine infusion (contractile reserve) is thought to be associated with a better prognosis than in patients without contractile reserve. It would be of interest to evaluate whether there are differences in plasma levels of natriuretic peptides in patients with heart failure with or without contractile reserve during low dose dobutamine infusion.

We therefore studied a cohort of patients referred for low dose dobutamine echocardiography and correlated the findings with their plasma concentrations of natriuretic peptides.

\section{METHODS}

\section{Patient population and study protocol}

The study population consisted of 66 consecutive patients who were referred for low dose dobutamine stress echocardiography for the evaluation of contractile reserve in areas of regional contractile dysfunction. The study population was divided into two groups on the basis of their left ventricular function and heart failure symptoms. Group 1 consisted of 31 patients with ischaemic cardiomyopathy (left ventricular ejection fraction $\leqslant 40 \%$ because of chronic coronary artery disease) and heart failure symptoms. Group 2 consisted of 35 patients with normal left ventricular function and no heart failure symptoms. Because the aim of the study was to evaluate the relation between plasma natriuretic peptides and contractile reserve in patients with ischaemic cardiomyopathy, those with primary cardiomyopathy, concomitant significant valvar disease, or left ventricular hypertrophy were excluded.

The study protocol was as follows:

- measurements of plasma levels of ANP and BNP using immunoradiometric assays;

- assessment of global and regional left ventricular function using resting cross sectional echocardiography;

- assessment of contractile reserve using low dose dobutamine stress echocardiography.

The local medical ethics committee approved the study protocol and all patients gave their informed consent.

\section{Cardiac peptide measurements}

Before stress echocardiography, blood samples were drawn from a peripheral vein, after the patient had rested for at least 30 minutes in a supine position. The blood samples were 
collected in prechilled tubes containing edetic acid (EDTA, $1.9 \mathrm{mg} / \mathrm{ml}$ ) and the protease inhibitor aprotonin (Trasylol, $100 \mathrm{kIU} / \mathrm{ml}$ ) to prevent breakdown of the cardiac peptides. Samples were stored on ice and promptly centrifuged at $3000 \mathrm{rpm}\left(4^{\circ} \mathrm{C}\right)$ for 10 minutes. The plasma was separated and stored at $-80^{\circ} \mathrm{C}$. Plasma concentrations of ANP and BNP were determined using standard commercially available immunoradiometric assay kits (Shionoria ANP and BNP kits, Shionogi, Osaka, Japan).

\section{Echocardiography}

We employed a commercially available imaging system (Hewlett Packard Sonos 5500, Andover, Massachusetts, USA) and a $1.8 \mathrm{MHz}$ transducer using second harmonic imaging to optimise endocardial border visualisation. Cross sectional imaging was done with the patient in the left lateral position, and standard views (in cine-loop format) were recorded on optical disk. Resting images were obtained first, followed by low dose dobutamine stress echocardiography. Dobutamine was given intravenously at a dose of $5 \mu \mathrm{g} / \mathrm{kg}$ body weight per minute for five minutes, followed by a $10 \mu \mathrm{g} / \mathrm{kg} / \mathrm{min}$ dose for five minutes.

\section{Assessment of the left ventricular ejection fraction} The left ventricular ejection fraction (LVEF) was determined off-line by the cross sectional biplane disk method using the modified Simpson's rule. ${ }^{9}$ The endocardial borders of the two and four chamber apical views were digitally traced at end diastole and end systole. Subsequently, left ventricular end diastolic and end systolic volumes were derived and the LVEF was calculated. An LVEF of $\leqslant 40 \%$ was considered indicative of severe left ventricular dysfunction.

\section{Assessment of contractile reserve}

Two experienced observers, unaware of the clinical data, scored the digitised echocardiograms off-line. In case of disagreement, a majority decision was achieved by a third observer.

The left ventricle was divided into 16 segments according to the American Society of Echocardiography. ${ }^{10}$ Regional wall motion and systolic wall thickening were scored using a five point grading scale: 1, normal; 2, mildly hypokinetic; 3, severely hypokinetic; 4, akinetic; 5, dyskinetic. Segments with severe hypokinesia, akinesia, or dyskinesia were considered abnormal. The presence of contractile reserve was defined as an improvement in segmental wall motion score by $\geqslant 1$ grade following infusion of low dose dobutamine $(5 \mu \mathrm{g} / \mathrm{kg} / \mathrm{min}$ or $10 \mu \mathrm{g} / \mathrm{kg} / \mathrm{min})$ in two or more severely dyssynergic segments.

\section{Statistical analysis}

Continuous data are expressed as mean (SD). Natriuretic peptide concentrations are presented as medians (SD). Percentages are rounded. Continuous variables are compared using the Student $t$ test for unpaired samples. Comparisons between the natriuretic peptide concentrations were done on $\log$ transformed concentrations. A probability value of $\mathrm{p}<0.05$ was considered significant.

\section{RESULTS}

\section{Patient characteristics}

The clinical characteristics of the total study population of 66 patients are presented in table 1 . In all, 31 patients had ischaemic cardiomyopathy, with an LVEF of $\leqslant 40 \%$ and heart failure symptoms. These comprised group 1. Their New York Heart Association (NYHA) functional class was on average 2.7 (0.6). Group 2 comprised 35 patients with normal left ventricular function and no heart failure symptoms.

\begin{tabular}{ll} 
Table 1 & \multicolumn{1}{c}{ Baseline characteristics } \\
\hline Men/women & $41(62) / 25(38)$ \\
Age (years) (mean (SD)) & $63(13)$ \\
Diabetes mellitus & $2(3)$ \\
Hypercholesterolaemia & $20(30)$ \\
Smoking & $8(12)$ \\
History & $19(29)$ \\
Myocardial infarction & $3(5)$ \\
Coronary angioplasty & $10(15)$ \\
Coronary bypass surgery & \\
Medical treatment & $20(30)$ \\
$\beta$ Blockers & $10(15)$ \\
Calcium channel blockers & $8(12)$ \\
Nitrates & $13(20)$ \\
ACE inhibitors & $8(12)$ \\
Diuretics & $5(8)$ \\
Digoxin & $26(39)$ \\
Aspirin & $20(30)$ \\
Cholesterol lowering drugs & \\
\hline \multicolumn{2}{l}{ Data are $n(\%)$ unless stated otherwise. } \\
ACE, angiotensin converting enzyme.
\end{tabular}

\section{Echocardiography}

The LVEF at rest in group 1 was 33 (7)\% compared with 60 $(9) \%$ in group $2(p<0.0001)$. Patients in group 1 had on average 8.1 (6.5) dysfunctional segments, $v$ 1.3 (3.7) in group $2(\mathrm{p}<0.0001)$. The wall motion score index was $2.2(0.9)$ in group 1 and $1.2(0.5)$ in group $2(p<0.0001)$. Low dose dobutamine stress echocardiography was undertaken in all patients without side effects. The haemodynamic changes in response to dobutamine are presented in table 2 . Contractile reserve was observed in 19 patients in group $1(61 \%)$ and in all the patients in group 2 .

\section{Natriuretic peptide concentrations}

The plasma concentrations of each natriuretic peptide were significantly raised in patients with abnormal left ventricular function. Figure 1 shows that the plasma ANP concentrations were higher in group 1 than in group 2, at 17.8 (32.8) $v 7.2$ (9.7) $\mathrm{pmol} / \mathrm{l}(\mathrm{p}<0.005)$. In line with this, plasma BNP concentrations were also higher in group l, at $24.4(69.0) v$ 5.0 (14.3) pmol/l $(\mathrm{p}<0.001)$. There was, however, substantial variation in individual ANP and BNP values in the patients in group 1 (fig 2).

\section{Natriuretic peptides and contractile reserve in patients with an LVEF of $\leqslant \mathbf{4 0 \%}$}

In group 1, the presence of contractile reserve was associated with lower plasma concentrations of natriuretic peptides: ANP, 14.2 (9.1) v 24.2 (44.2) pmol/l, p < 0.05 (fig 3); BNP, $20.2(25.5) \vee 37.5(93.8) \mathrm{pmol} / \mathrm{l}, \mathrm{p}<0.05$.

\section{DISCUSSION}

Previous studies have shown that plasma natriuretic peptide concentrations are raised in patients with congestive heart failure; moreover, raised levels of these peptides are

\begin{tabular}{|c|c|c|c|}
\hline & \multirow[b]{2}{*}{ Baseline } & \multicolumn{2}{|c|}{ Dobutamine infusion } \\
\hline & & $5 \mu \mathrm{g} / \mathrm{kg} / \mathrm{min}$ & $10 \mu \mathrm{g} / \mathrm{kg} / \mathrm{min}$ \\
\hline $\begin{array}{l}\text { Heart rate (beats/min) } \\
\text { Systolic BP ( } \mathrm{mmHg}) \\
\text { Diastolic BP (mmHg) } \\
\text { Rate-pressure product }\end{array}$ & $\begin{array}{c}74(15) \\
131(22) \\
76(12) \\
9595(2446)\end{array}$ & $\begin{aligned} 81(22)^{*} \\
132(25) \\
75(13) \\
10554(3039)\end{aligned}$ & $\begin{array}{l}90(26)^{*} \dagger \\
131(23) \\
72(11)^{*} \\
11693(3862) \dagger\end{array}$ \\
\hline \multicolumn{4}{|c|}{$\begin{array}{l}\text { Values are mean (SD). } \\
{ }^{*} \mathrm{p}<0.05 v \text { baseline; } \uparrow \mathrm{tp}<0.05 \vee 5 \mu \mathrm{g} / \mathrm{kg} / \mathrm{min} \text { dobutamine infusion. } \\
\mathrm{BP} \text {, blood pressure. }\end{array}$} \\
\hline
\end{tabular}




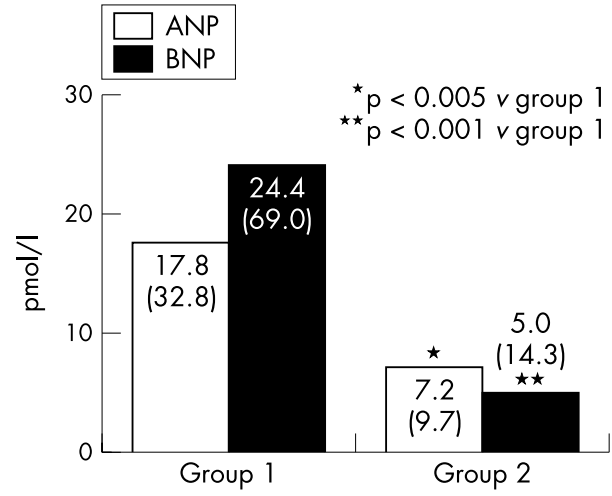

Figure 1 Plasma concentrations of atrial natriuretic peptide (ANP) and brain natriuretic peptide (BNP) in group 1 and group 2.

associated with a poor prognosis..$^{4-8}$ In the current study, we found that patients with severe left ventricular dysfunction had markedly raised plasma concentrations of natriuretic peptides, although individual data varied substantially. In group 1 (LVEF $\leqslant 40 \%$ ), comparison of patients with and without contractile reserve showed that those with no reserve had significantly higher plasma levels of natriuretic peptides and may be at higher risk of future cardiac events. The natriuretic peptides play a role protecting the heart from volume overload. ${ }^{11}$ The cardiac hormones ANP and BNP are produced in the atria and ventricles, respectively, in response to an increase in wall stretch or pressure. Raised plasma ANP and BNP concentrations have a natriuretic and diuretic effect. In addition, a high plasma BNP causes a fluid shift from the capillary bed to the interstitium, thereby decreasing preload and blood pressure. Hence, ANP and BNP are functional counterparts of the renin-angiotensin-aldosterone system. Accordingly, the plasma levels of these peptides have been used to diagnose patients with heart failure. ${ }^{12}$ In the present study, the plasma ANP and BNP concentrations were significantly raised in the subset of patients with a severely depressed LVEF.

In patients with ischaemic cardiomyopathy, the presence of contractile reserve is related to the extent of viable tissue. ${ }^{13}$ Histopathological analysis of biopsies taken (during surgical revascularisation) from segments with and without contractile reserve showed that a higher percentage of fibrosis was present in the segments without contractile reserve. ${ }^{14}{ }^{15}$ In

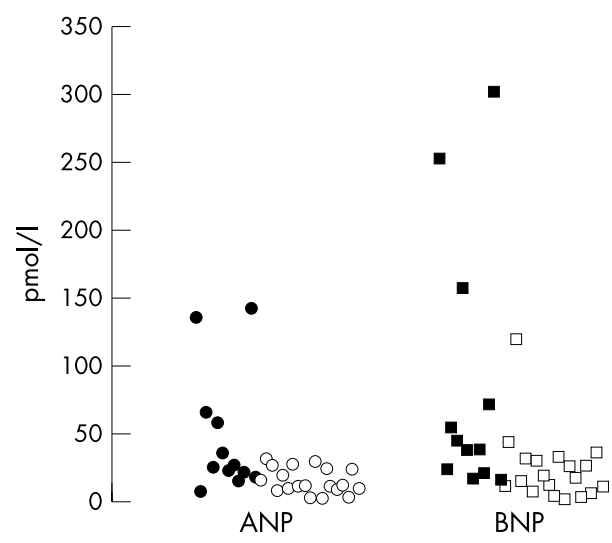

Figure 2 Scatterplot showing the individual values for atrial natriuretic peptide (ANP) and brain natriuretic peptide (BNP) in the patients with ischaemic cardiomyopathy and heart failure symptoms, with (white circles and white squares) and without (black circles and black squares) contractile reserve (group 1).

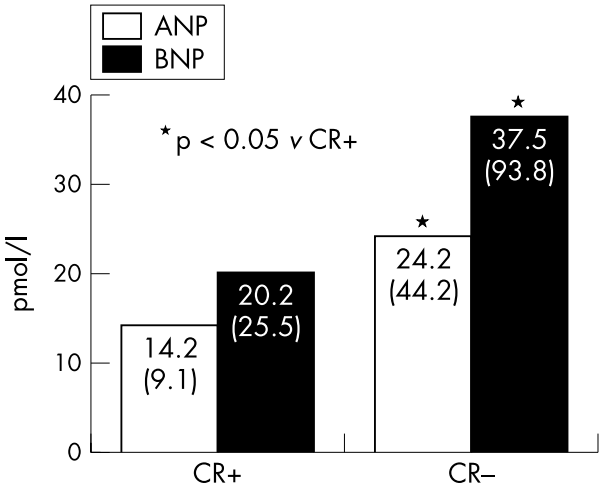

Figure 3 Mean plasma atrial natriuretic peptide (ANP) and brain natriuretic peptide (BNP) concentrations in group 1, in patients with and without preserved left ventricular myocardial contractile reserve.

studies assessing prognosis, the presence of contractile reserve has been shown to have a positive influence on long term outcome. ${ }^{16}{ }^{17}$

It is of interest that in the current study, patients with contractile reserve had significantly lower plasma concentrations of natriuretic peptides than patients without contractile reserve. Accordingly, both prognostic indices appear to point in the same direction. From the current study, the precise interrelations between these two variables cannot be derived, and further studies are needed to provide insight in the pathophysiology underlying our observations. However, the combined assessment of (high plasma levels of) natriuretic peptides and (absence of) contractile reserve may help to identify patients at relatively high risk for future events.

\section{Authors' affiliations}

A F L Schinkel, E C Vourvouri, M Bountioukos, V Rizzello, E Biagini, E Agricola, A Elhendy, J R T C Roelandt, D Poldermans, Department of Cardiology, Thoraxcentre, Erasmus Medical Centre, Rotterdam,

Netherlands

F Boomsma, Department of Internal Medicine, Erasmus Medical Centre J J Bax, Department of Cardiology, Leiden University Medical Centre, Leiden, Netherlands

\section{REFERENCES}

1 McMurray JJ, Stewart S. Heart failure: epidemiology, aetiology, and prognosis of heart failure. Heart 2000;83:596-602.

2 McCullough PA, Philbin EF, Spertus JA, et al. Confirmation of a heart failure epidemic: findings from the Resource Utilization Among Congestive Heart Failure (REACH) study J Am Coll Cardiol 2002:39:60-9.

3 Nielsen OW, Hilden J, Larsen CT, et al. Cross sectional study estimating prevalence of heart failure and left ventricular systolic dysfunction in community patients at risk. Heart 2001;86:172-8.

4 Nakamura $M$, Endo $H$, Nasu $M$, et al. Value of plasma B type natriuretic peptide measurement for heart disease screening in a Japanese population. Heart 2002;87:131-5.

5 Cowie MR, Struthers AD, Wood DA, et al. Value of natriuretic peptides in assessment of patients with possible new heart failure in primary care. Lancet 1997; 350:1349-53.

6 Omland T, Akvaag A, Vik-Mo H, et al. Plasma cardiac natriuretic peptide determination as a screening test for the detection of patients with mild left ventricular impairment. Heart 1996;76:232-7.

7 McDonagh TA, Robb SD, Murdoch DR, et al. Biochemical detection of leftventricular systolic dysfunction. Lancet 1998;351:9-13.

8 McDonagh TA, Cunningham AD, Morrison CE, et al. Left ventricular dysfunction, natriuretic peptides, and mortality in an urban population. Heart $2001 ; 86: 21-6$

9 Schiller NB, Shah PM, Crawford M, et al. Recommendations for quantitation of the left ventricle by two-dimensional echocardiography. J Am Soc Echocardiogr 1989;2:358-67.

10 Bourdillon PD, Broderick TM, Sawada SG, et al. Regional wall motion index for infarct and non-infarct region after reperfusion in acute myocardial infarction: comparison with global wall motion index. J Am Soc Echocardiogr 1989;2:398-407

11 Schrier RW, Abraham WT. Hormones and hemodynamics in heart failure N Engl J Med 1999;341:577-85. 
12 McCullough PA, Nowak RM, McCord J, et al. B-type natriuretic peptide and clinical judgment in emergency diagnosis of heart failure: analysis from Breathing Not Properly (BNP) Multinational Study. Circulation 2002;106:416-22

13 Lombardo A, Loperfido F, Trani C, et al. Contractile reserve of dysfunctional myocardium after revascularization: a dobutamine stress echocardiography study. J Am Coll Cardiol 1997;30:633-40.

14 Panza JA, Dilsizian V, Curiel RV, et al. Myocardial blood flow at rest and contractile reserve in patients with chronic coronary artery disease and left ventricular dysfunction. J Nucl Cardiol 1999;6:487-94.
15 Maes A, Flameng W, Nuyts J, et al. Histological alterations in chronically hypoperfused myocardium. Correlation with PET findings. Circulation 1994;90:735-45

16 Picano E, Sicari R, Landi $P$, et al. Prognostic value of myocardial viability in medically treated patients with global left ventricular dysfunction early after an acute uncomplicated myocardial infarction: a dobutamine stress echocardiographic study. Circulation 1998;98:1078-84.

17 Carlos ME, Smart SC, Wynsen JC, et al. Dobutamine stress echocardiography for risk stratification after myocardial infarction. Circulation 1997:95:1402-10.

\section{IMAGES IN CARDIOLOGY}

\section{Dynamic respiratory changes in hypertrophic cardiomyopathy}

A 50 year old woman presented with severe exertional dyspnoea. On physical examination, she had a 3/6 systolic ejection murmur that virtually disappeared with inspiration. Transthoracic echocardiography demonstrated findings typical of hypertrophic cardiomyopathy with a pronounced increase in ventricular septal thickness and an outflow gradient of $100 \mathrm{~mm} \mathrm{Hg}$. The patient underwent cardiac catheterisation (panel below: Ao, aortic pressure, LA, left atrial pressure, LV, left ventricular pressure, PA, pulmonary artery pressure). In the resting state, there was a significant outflow tract gradient between the left ventricular and aortic pressure. However, with each inspiration, there was a large decrease in the left ventricular outflow tract gradient. The "spike and dome" morphology of the aortic pressure curve virtually disappeared during peak inspiration, indicating a notable resolution of the outflow obstruction.

The pronounced decrease in the intensity of the systolic murmur with inspiration is unique to hypertrophic cardiomyopathy, secondary to the dynamic nature of the obstruction. The left ventricular outflow tract obstruction in patients with hypertrophic cardiomyopathy is highly dependent upon preload, afterload, and contractility. With inspiration, the intrathoracic and intrapericardial pressures decrease to a greater extent than the intracardiac pressures. As a result, the left ventricular transmural pressure increases, increasing the afterload on the left ventricle, thus decreasing the obstruction and the left ventricular outflow tract gradient.

The dynamic changes of the murmur intensity with respiration may help in the differential diagnosis of a systolic murmur. In patients with hypertrophic cardiomyopathy, there may be a notable decrease in the intensity of the murmur during peak inspiration. Since the outflow tract obstruction is also decreased, there may even be an increase in arterial pressure, which has been called "reverse pulsus paradoxus". In contrast, patients with right sided murmurs will have an increase in the intensity of the murmur with inspiration. In patients with murmurs of fixed outflow tract obstruction or mitral regurgitation, there may be a slight decrease in the intensity of the murmur with inspiration from a slight decrease in preload but the aortic pressure will also decrease.

E S Brilakis R A Nishimura rnishimura@mayo.edu

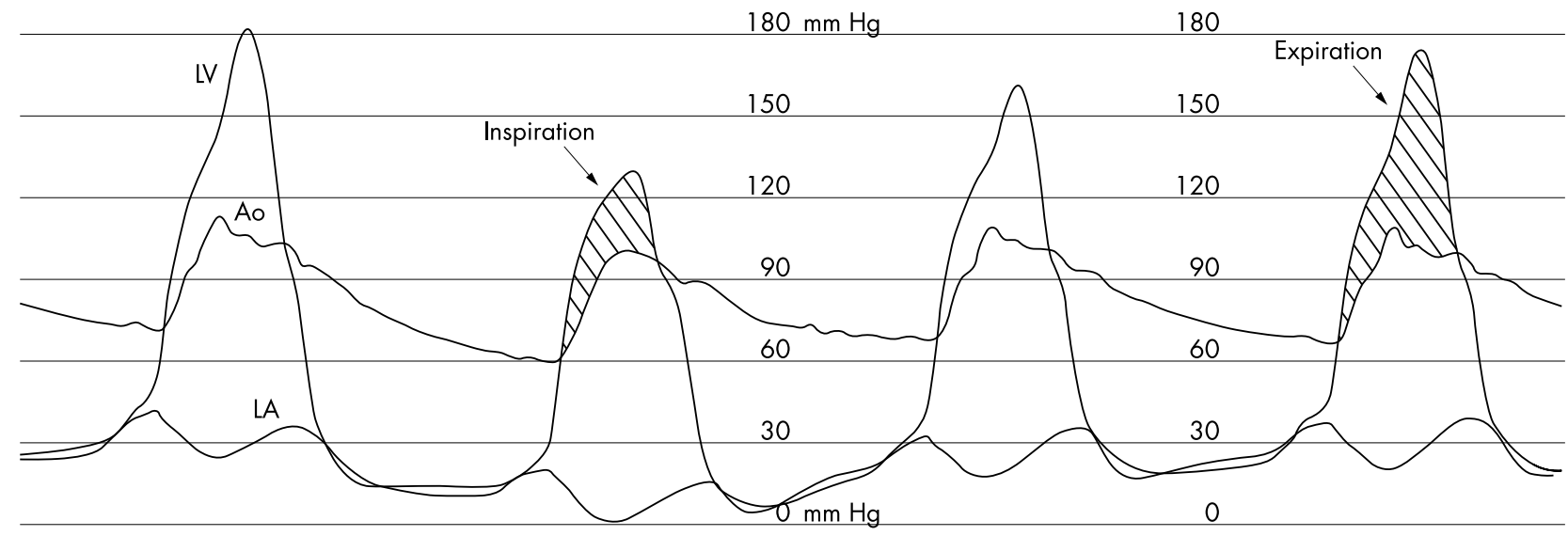

